\title{
Realization of center symmetry in two adjoint flavor large-N Yang-Mills
}

\author{
Simon Catterall, ${ }^{a}$ Richard Galvez ${ }^{a}$ and Mithat Ünsal ${ }^{b}$ \\ ${ }^{a}$ Department of Physics, Syracuse University, \\ Syracuse, NY 13244, U.S.A. \\ ${ }^{b}$ SLAC and Physics Department, Stanford University, \\ Stanford, CA 94305, U.S.A. \\ E-mail: smc@phy.syr.edu, ragalvez@syr.edu, unsal@slac.stanford.edu
}

ABSTRACT: We report on the results of numerical simulations of $\mathrm{SU}(N)$ lattice Yang Mills with two flavors of (light) Wilson fermion in the adjoint representation. We analytically and numerically address the question of center symmetry realization on lattices with $\Gamma$ sites in each direction in the large- $N$ limit. We show, by a weak coupling calculation that, for massless fermions, center symmetry realization is independent of $\Gamma$, and is unbroken. Then, we extend our result by conducting simulations at non zero mass and finite gauge coupling. Our results indicate that center symmetry is intact for a range of fermion mass in the vicinity of the critical line on lattices of volume $2^{4}$. This observation makes it possible to compute infinite volume physical observables using small volume simulations in the limit $N \rightarrow \infty$, with possible applications to the determination of the conformal window in gauge theories with adjoint fermions.

Keywords: Lattice Gauge Field Theories, 1/N Expansion

ARXiv EPRINT: 1006.2469 


\section{Contents}

1 Introduction 1

2 Lattice action and methods 2

3 Weak coupling analysis of center symmetry on $\Gamma^{4}$ lattice 3

4 Numerical results 5

$\begin{array}{lll}5 & \text { Conclusions } & 10\end{array}$

\section{Introduction}

Large- $N$ gauge theories compactified on a torus have properties independent of the compactification radii provided $i$ ) center symmetry and ii) translation symmetry are not spontaneously broken [1-5]. We refer to this property as large- $N$ volume independence. In a lattice regularized theory, the reduction to a one-site model is known as "Eguchi-Kawai (EK) or large- $N$ reduction". Volume independence is a property of both confining and conformal field theories provided the necessary symmetries are satisfied $[6,7]$.

If valid, large- $N$ reduction may have practical benefits in lattice gauge theory. For example, the computational cost of simulating a $L^{4}$ lattice (excluding the effects of critical slowing down) grows as $L^{5}$ while the computational cost of simulating at large- $N$ naively grows only as $N^{\frac{7}{2}}$. These observations are particularly important in theories with large finite volume effects such as conformal or near conformal theories. One such example of current interest is the minimal walking theory (MWT) which has been proposed as a technicolor model allowing for a dynamical breaking of electroweak symmetry [8-13]. This model employs two Dirac (four Weyl) flavors of adjoint fermion in an SU(2) gauge theory and is thought to be conformal or near conformal in the infrared. A near conformal behavior is believed to be a necessary ingredient for constructing a realistic "walking" gauge theory capable of breaking the electroweak symmetry of the Standard Model while respecting the bounds on new physics implied by electroweak precision measurements at LEP. Because of these features, this model has been studied extensively in recent years by the lattice community [14-23].

For four dimensional confining gauge theories, it was usually believed that large- $N$ reduction should be valid only above a critical size, $L>L_{\mathrm{c}}$, below which center symmetry would break spontaneously invalidating the equivalence. For example, for pure YM theory, $L_{\mathrm{c}} \sim \Lambda^{-1}$ is about a few fermi [24]. This understanding changed in recent years [5, 25, 26]. Ref. [5] has shown that Yang-Mills theory with multiple adjoint fermions [QCD(adj)], where the fermions are endowed with (non-thermal) periodic boundary conditions, satisfies 
volume independence down to arbitrarily small volumes. Another way to have a working volume independence is to use double-trace deformations, by explicitly adding the modulus square of Wilson line operators [25]. This suppresses the unwanted breaking, without altering the leading large- $N$ dynamics of the theory. In this sense, the deformed theory also overcomes the $L_{\mathrm{c}}$ "impasse" of pure theory. ${ }^{1}$

Switching back to the minimal walking theory, one of the most pressing questions to resolve using non-perturbative studies is to clarify whether this model does indeed lie within the so-called conformal window or whether it lies just outside this window and perhaps can serve as an example of a walking gauge theory. To distinguish conformal from near conformal behavior it would seem that very large volume simulations would be necessary. Furthermore, since the conformal window for models with adjoint fermions is expected, at leading order in $N$, to be independent of $N$, the question can equally be phrased in a general $N_{\mathrm{f}}$-flavor $\mathrm{SU}(N)$ gauge theory. Continuum analysis indicates that this class of theories, endowed with periodic boundary conditions, obey volume independence, regardless of their long-distance behavior. Thus, the question of whether $N_{\mathrm{f}}=2$ theory is conformal or confining can be addressed on small $2^{4}$ or even $1^{4}$ lattices [6]. Since volume reduction depends on the property of center symmetry it is important to see, nonperturbatively, whether the large- $N$ theory is center symmetric in the limit of small volumes. This examination will be the main goal of this work.

As mentioned above, it is well known that center symmetry spontaneously breaks in large- $N$ pure Yang Mills theory for dimensions greater than two [3, 24]. However, a one-loop continuum analysis on $R^{3} \times S^{1}$ (which generalizes to arbitrary toroidal compactifications) has shown that the center symmetry will be restored if the theory is coupled to one or more flavors of (light or massless) adjoint ${ }^{2}$ Weyl fermion [5]. See, also refs. [31-37] for related work.

Ref. [5] also conjectured that a single-site version of $\mathrm{QCD}(\mathrm{Adj})$, which is just the EK matrix model augmented with adjoint representation Grassmann variables, will reproduce the leading large- $N$ behavior of all expectation values, and zero-momentum connected correlators of single-trace observables in infinite volume QCD(Adj). This conjecture received strong numerical confirmation in single flavor simulations in [26] for Wilson fermions, in one-loop lattice perturbative analysis as in [38] using overlap fermions, and in a one-loop analysis and simulations using the one-site theory with Wilson fermions in [39].

Our goal in this work is to extend the one flavor result of [26], to two flavors in preparation for studies of the conformal window in the MWT theory.

\section{Lattice action and methods}

We employed a $2^{4}$ lattice volume in our work. The reason we do so is to take advantage of both volume and $N$ scaling simultaneously, as we are working with relatively smaller

\footnotetext{
${ }^{1}$ Earlier modifications known as quenched - [3] and twisted — EK models [4] have recently been shown to fail due to nonperturbative effects [27-30].

${ }^{2}$ With fundamental fermions, center symmetry is explicitly broken, but it still is an approximate symmetry in the $N_{\mathrm{f}}=$ fixed as $N \rightarrow \infty$ limit. Volume independence is valid in the confined phase of such a theory. This domain can be extended to arbitrarily small radii by the addition of double-trace deformations [25].
} 
$N$ than that used in $[26,39]$. The lattice action we employ consists of the usual Wilson plaquette term

$$
S_{G}=-\frac{\beta}{2} \sum_{x} \sum_{\mu>\nu} \operatorname{Re} \operatorname{Tr}\left(U_{\mu}(x) U_{\nu}(x+\mu) U_{\mu}^{\dagger}(x+\nu) U_{\nu}^{\dagger}(x)\right)
$$

together with the Wilson action for two Dirac quarks in the adjoint representation

$$
\begin{aligned}
S_{F}= & -\frac{1}{2} \sum_{x} \sum_{\mu} \bar{\psi}(x)\left(V_{\mu}(x)\left(I-\gamma_{\mu}\right) \psi(x+\mu)+V_{\mu}^{\dagger}(x-\mu)\left(I+\gamma_{\mu}\right) \psi(x-\mu)\right) \\
& +\sum_{x}(m+4) \bar{\psi}(x) \psi(x)
\end{aligned}
$$

where the symmetric links are given by

$$
V_{\mu}^{a b}(x)=\operatorname{Tr}\left(S^{a} U_{\mu}(x) S^{b} U_{\mu}^{T}(x)\right),
$$

and the matrices $S^{a}, a=1,2,3$ are the usual Pauli matrices. We use the usual HMC algorithm [40] to simulate this model at a variety of 't Hooft couplings $\lambda=g^{2} N$ and bare quark masses $m$. Periodic boundary conditions are used for all lattice directions.

\section{Weak coupling analysis of center symmetry on $\Gamma^{4}$ lattice}

Before showing the results of our numerical simulations, it is instructive to consider the question of center symmetry realization in a weak coupling lattice perturbation theory analysis. We consider a four-dimensional lattice with $\Gamma^{4}$ sites, labeled as $L^{\Gamma}$. Generalization to asymmetric lattices with $\Gamma_{\mu}$ sites in the $\mu$-th directions is obvious. Let us label the Wilson line (none of the circles is thermal, with a slight abuse of language, we use the Wilson and Polyakov line interchangeably) along the $\mu$-th direction as $P_{\mu}$, given by

$$
P_{\mu}(x)=U_{\mu}(x) U_{\mu}\left(x+e_{\mu}\right) U_{\mu}\left(x+2 e_{\mu}\right) \ldots U_{\mu}\left(x+(\Gamma-1) e_{\mu}\right)
$$

$P_{\mu}$ is gauge covariant and its trace is gauge invariant. The space of classical vacua is parameterized as a space of commuting (diagonal) Wilson lines, $\left[P_{\mu}, P_{\nu}\right]=0$ :

$$
P_{\mu}=\operatorname{Diag}\left(e^{i \theta_{\mu}^{1}}, e^{i \theta_{\mu}^{2}}, \ldots, e^{i \theta_{\mu}^{N}}\right)
$$

In the weak coupling regime, it is easy to evaluate the one-loop potential by integrating out the heavy modes. Let us first find the spectrum of gauge and fermionic fluctuations in the background of commuting Wilson lines. Our calculation generalizes section 2 of ref. [35].

The spectrum of gauge fluctuations in the background of commuting Wilson lines is

$$
M_{g}^{2}\left(k_{\mu}, \theta_{\mu}^{i j}\right)=\sum_{\mu=1}^{4}\left[\frac{2}{a} \sin \left(\frac{2 \pi k_{\mu}+\theta_{\mu}^{i j}}{2 \Gamma}\right)\right]^{2}, \quad k_{\mu}=1, \ldots \Gamma, \quad i, j=1, \ldots N
$$


where $\theta_{\mu}^{i j} \equiv \theta_{\mu}^{i}-\theta_{\mu}^{j}$. And analogously, for Wilson fermions with bare mass $m$ and Wilson parameter $r=1$, the spectrum of fermionic fluctuations is given by

$$
M_{f}^{2}\left[k_{\mu}, \theta_{\mu}^{i j}, m\right]=\frac{1}{a^{2}} \sum_{\mu=1}^{4} \sin ^{2}\left(\frac{2 \pi k_{\mu}+\theta_{\mu}^{i j}}{\Gamma}\right)+\left[m+\frac{2}{a} \sum_{\mu=1}^{4} \sin ^{2}\left(\frac{2 \pi k_{\mu}+\theta_{\mu}^{i j}}{2 \Gamma}\right)\right]^{2}
$$

Both of these formulas are quite intuitive: Setting $\theta_{\mu}^{i j}=0$ gives the usual spectrum of gauge bosons and Wilson-fermions (with $r=1$ ) in lattice gauge theory. Setting $\Gamma=1$, the bosonic formula gives the distance between the eigenvalues of the Wilson line. The fermionic one is similar, but differs due to the fermion dispersion relation on the lattice. Note that lattice momenta $k_{\mu}$ and eigenvalue difference $\theta_{\mu}^{i j}$ appear on the same footing.

The one-loop action induced by bosonic and fermionic fluctuations on $L^{\Gamma}$ yields

$$
S_{1-\text { loop }}\left[\Gamma, \theta_{\mu}^{i j}, m\right]=2 \sum_{i<j} \sum_{\vec{k} \in L^{\Gamma}} \log \left(M_{g}^{2}\left(k_{\mu}, \theta_{\mu}^{i j}\right)\right)-4 N_{f} \sum_{i<j} \sum_{\vec{k} \in L^{\Gamma}} \log \left(M_{f}^{2}\left(k_{\mu}, \theta_{\mu}^{i j}, m\right)\right)
$$

This formula captures all the interesting limits, including the 1-site lattice theory (EKversion of $\mathrm{QCD}(\operatorname{adj}))$ and the $\Gamma=\infty$ infinite lattice limit, as well as the continuum limit. Below, we concentrate on the case where the bare mass is set to zero, $m=0$.

For $N_{f}=0 \Gamma=1$ we recover the result of ref. [3] in which a spontaneous breaking of center symmetry occurs. If we take the continuum limit by using the usual scaling,

$$
\Gamma \rightarrow \infty, \quad a \rightarrow 0, \quad L=\Gamma a=\text { fixed }
$$

with $L \Lambda_{\mathrm{YM}} \ll 1\left(\Lambda_{\mathrm{YM}}\right.$ is the strong-coupling scale of the theory), one must reproduce the continuum one-loop result from perturbation theory. Indeed, using

$$
\lim _{a \rightarrow 0} \frac{2}{a} \sin \left(\frac{2 \pi k_{\mu}+\theta_{\mu}^{i j}}{2 \Gamma}\right)=\left(2 \pi k_{\mu}+\theta_{\mu}^{i j}\right) L^{-1},
$$

the one-loop potential in pure YM theory on continuum small $T^{4}$ is produced. This expression can equivalently be written by using Poisson resummation in terms of gauge invariant Wilson lines and the result is $S_{1-\text { loop }}^{\text {YM }}\left[P_{\mu}\right]=-\frac{1}{\pi^{2}} \sum_{\vec{n} \in \mathbb{Z}^{4} \backslash\{0\}} \frac{1}{|\vec{n}|}\left|\operatorname{Tr}\left(P_{1}^{n_{1}} \ldots P_{4}^{n_{4}}\right)\right|^{2}$. Thus for Yang-Mills theory on small continuum $T^{4}$, since the masses of Wilson lines are all negative, the center symmetry is broken.

The case of $\Gamma=1$ and $N_{f} \geq 0.5$ is the full EK reduced version of QCD(adj) simulated in $[26,39]$. As opposed to gauge fluctuations which generate eigenvalue attraction in any domain where one-loop analysis is reliable, the adjoint fermions induce eigenvalue repulsion. This is the adjoint fermion-induced center-stabilization mechanism of ref. [5]. If we take the (naive) continuum limit by using the scaling (3.6), then the result reduces to

$$
S_{1-\text { loop }}^{\mathrm{QCD}}\left[P_{\mu}\right]=\left(1-2 N_{f}\right) S_{1-\text { loop }}^{\mathrm{YM}}\left[P_{\mu}\right]=\frac{1}{\pi^{2}} \sum_{\vec{n} \in \mathbb{Z}^{4} \backslash\{\mathbf{0}\}} \frac{\left(-1+2 N_{f}\right)}{|\vec{n}|^{4}}\left|\operatorname{Tr}\left(P_{1}^{n_{1}} \ldots P_{4}^{n_{4}}\right)\right|^{2}
$$

in agreement with ref. [5]. This implies, in the massless continuum limit, that all Wilson lines have positive masses and the center symmetry is unbroken. 


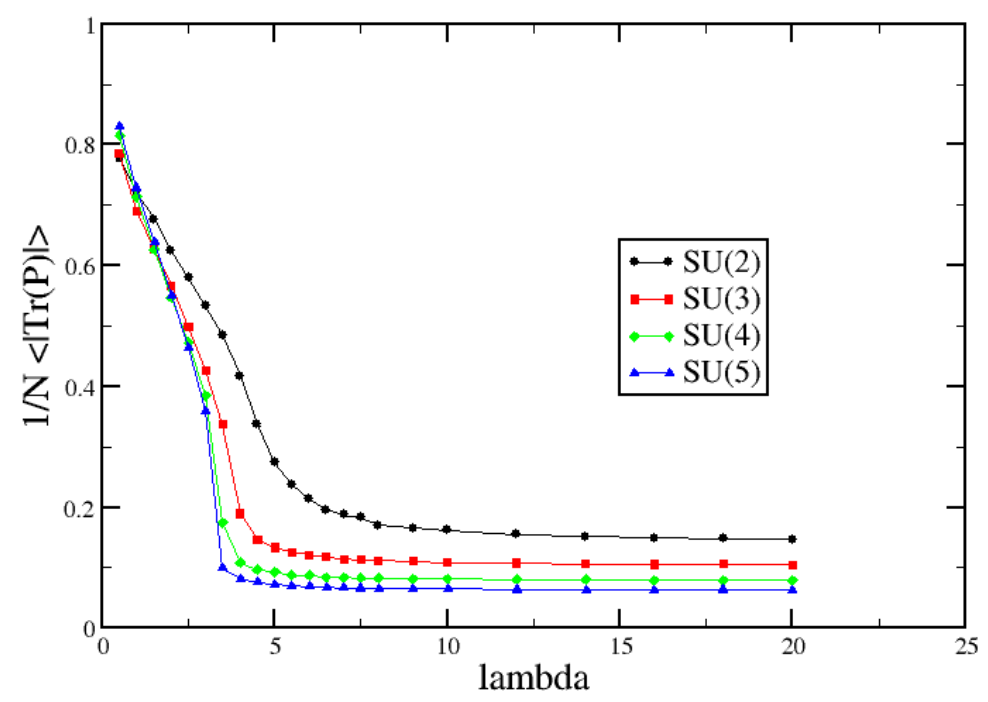

Figure 1. Simple Polyakov line in the quenched theory vs 't Hooft coupling $\lambda$.

For arbitrary $\Gamma$, the Poisson resummation (which can be done numerically) leads to an analog of the one-loop action (3.8). This yields, for massless fermions, that the center symmetry realization is independent of $\Gamma$, and is unbroken.

The above analysis is at weak coupling in lattice perturbation theory. In the next section, we would like to check that the conclusion regarding unbroken center symmetry can be generalized to finite couplings where a perturbative analysis is inapplicable, and non-zero fermion mass.

\section{Numerical results}

To look for a breaking of center symmetry we monitored a sequence of order parameters corresponding to correlators of Polyakov lines in different directions. The Polyakov line is defined in the usual way as in $(3.1),{ }^{3}$ while the line correlators we measure are given by

$$
\begin{aligned}
& M_{\mu \nu}^{(1)}=P_{\mu}(x) P_{\nu}(x) \\
& M_{\mu \nu}^{(2)}=P_{\mu}(x) P_{\nu}^{\dagger}(x)
\end{aligned}
$$

It is interesting to consider first the quenched model (with infinitely heavy fermions) ${ }^{4}$ in order to contrast the behavior of the theory coupled to dynamical adjoint fermions. Figure 1 shows a plot of the ensemble average of the absolute value of $\frac{1}{N} \operatorname{Tr}\left(P_{1}\right)$. The data is plotted as a function of the 't Hooft coupling $\lambda$ for values of $N=2-5$. Clearly for $\lambda>\lambda_{c} \sim 3.0$ the curves fall towards the $\mathrm{x}$-axis with larger $N$ consistent with a vanishing of the expectation value of the line $<\frac{1}{N} \operatorname{Tr}\left(P_{\mu}\right)>=0$ in the large $N$ limit. This implies that

\footnotetext{
${ }^{3}$ We average this quantity over all 8 points in the volume orthogonal to the line direction $\mu$.

${ }^{4}$ Here, and throughout the text, we use the word quenched to denote the theory without dynamical fermions. This is different from Quenched-EK [3] which refers to the freezing of the eigenvalues at the roots of unity.
} 


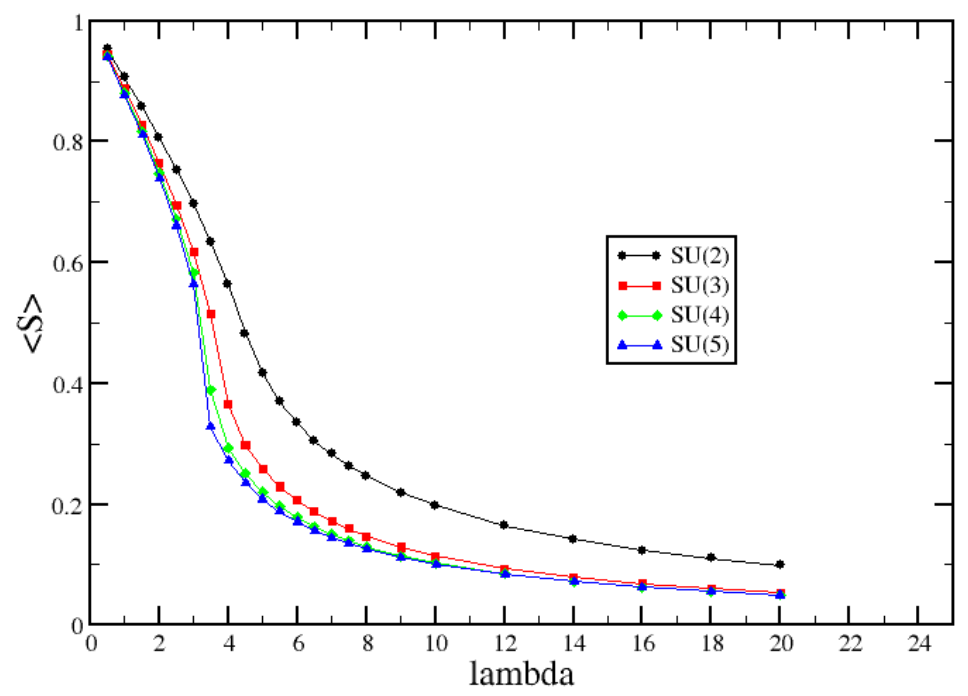

Figure 2. Plaquette action in the quenched theory vs 't Hooft coupling.

center symmetry remains unbroken at large $N$. However, a different behavior is seen for small $\lambda<\lambda_{c}$. In this region the curves for different $N$ all approach a fixed $N$-independent function $f(\lambda)$ for large $N$. Such a behavior indicates a spontaneous breaking of center symmetry. This two phase structure is also seen in the mean plaquette action which is plotted in figure 2 .

These results reproduce what has long been known for the pure YM theory: the lattice theory exists in two phases; a strong coupling lattice phase with unbroken center symmetry and a weak coupling phase in which the symmetry is spontaneously broken. These two phases are thought to be separated by a phase transition - the Gross-Witten transition which is hinted at by a potential discontinuity which appears to develop in the mean action for large $N$ as seen in figure 2. Continuum physics can be only obtained in the weak coupling phase and hence volume reduction is invalid in the continuum limit of the theory.

This behavior should be contrasted with the results for the theory with 2 flavors of dynamical adjoint fermions. Figure 3 shows a plot of the absolute value of the Polyakov line $\frac{1}{N}\left|\operatorname{Tr}\left(P_{1}\right)\right|$ for fixed 't Hooft coupling $\lambda=0.5$ as a function of the bare quark mass for a range of $N=2-6$. Notice that $\lambda=0.5$ lies well within the weak coupling phase of the quenched model - a regime in which the quenched model exhibits strong breaking of center symmetry. The adjoint fermions seem to strongly suppress the Polyakov line and this effect increases with larger $N$ consistent with the presence of exact center symmetry at large $N$. In figure 4 we show also for this same coupling the "pion mass" as give by the logarithm of the ratio of the pion correlator at zero and one unit of time separation $m_{\pi}=\log \frac{G_{\pi}(0)}{G_{\pi}(1)} \cdot{ }^{5}$ We expect the critical line to be located close to the minimum pion mass.

\footnotetext{
${ }^{5}$ This should not be taken seriously as the real pion mass in this theory which can only be extracted at large Euclidean times. But its value does correlate with the position of the critical line in the model. An equally good observable would be the number of conjugate gradient iterations needed to invert the Dirac operator which yields a very similar behavior with bare quark mass.
} 


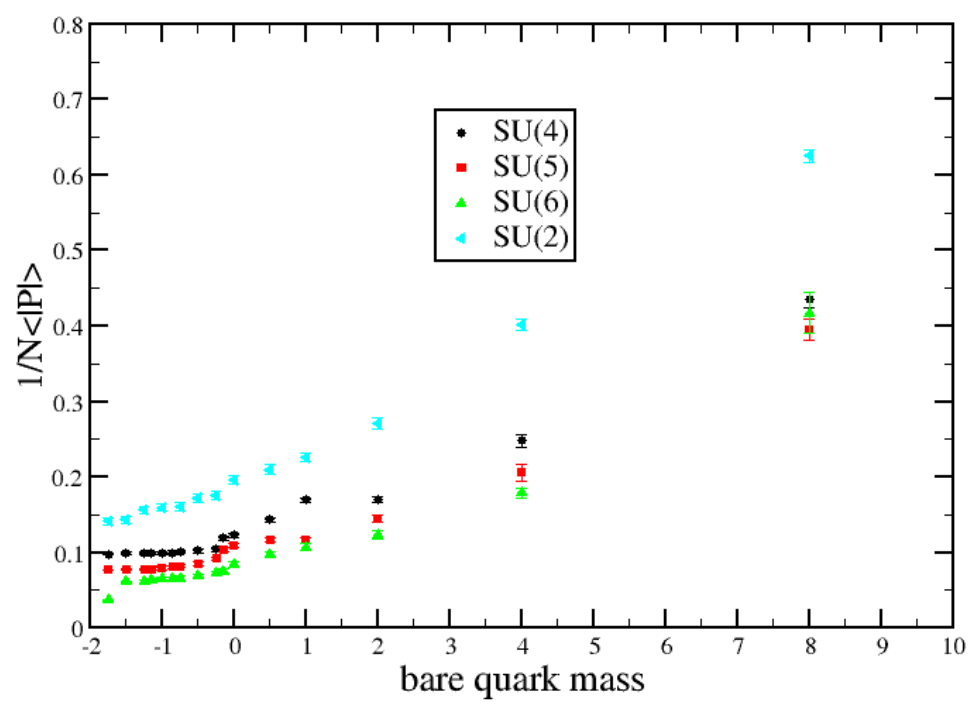

Figure 3. Polyakov line vs bare quark mass for 't Hooft coupling $\lambda=0.5$.

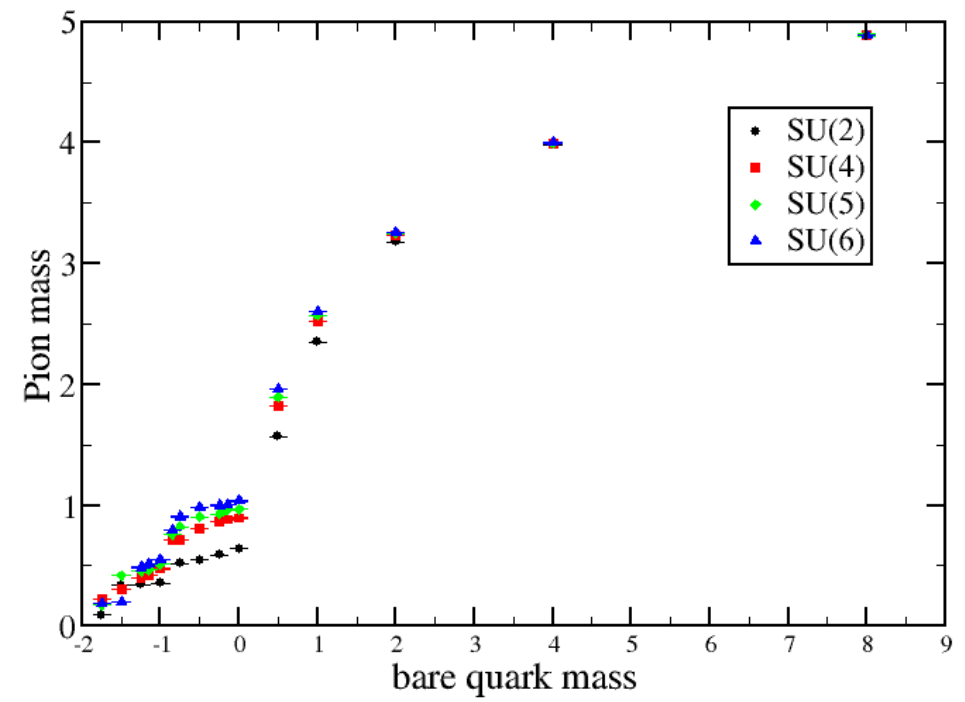

Figure 4. Pion mass vs bare quark mass for 't Hooft coupling $\lambda=0.5$.

By comparing these two plots it should be clear that for moderately light quark masses the magnitude of the Polyakov line falls with increasing $N$ consistent with a vanishing of the expectation value of $P_{\mu}$ in the limit $N \rightarrow \infty$. A more quantitative measure of this is given in figure 5 which plots the expectation value of the Polyakov line at $\lambda=0.5$ and bare quark mass $m=-1$ as a function of $1 / N$. A linear fit to the largest $N$ points yields an intercept which is consistent with zero and a restoration of center symmetry in the large $N$ limit. Further evidence in favor of center symmetry restoration comes from comparing a scatter plot of the expectation values of the trace of the Polyakov line in the complex plane in both quenched and dynamical cases at $\lambda=0.5$ (in the latter case the 


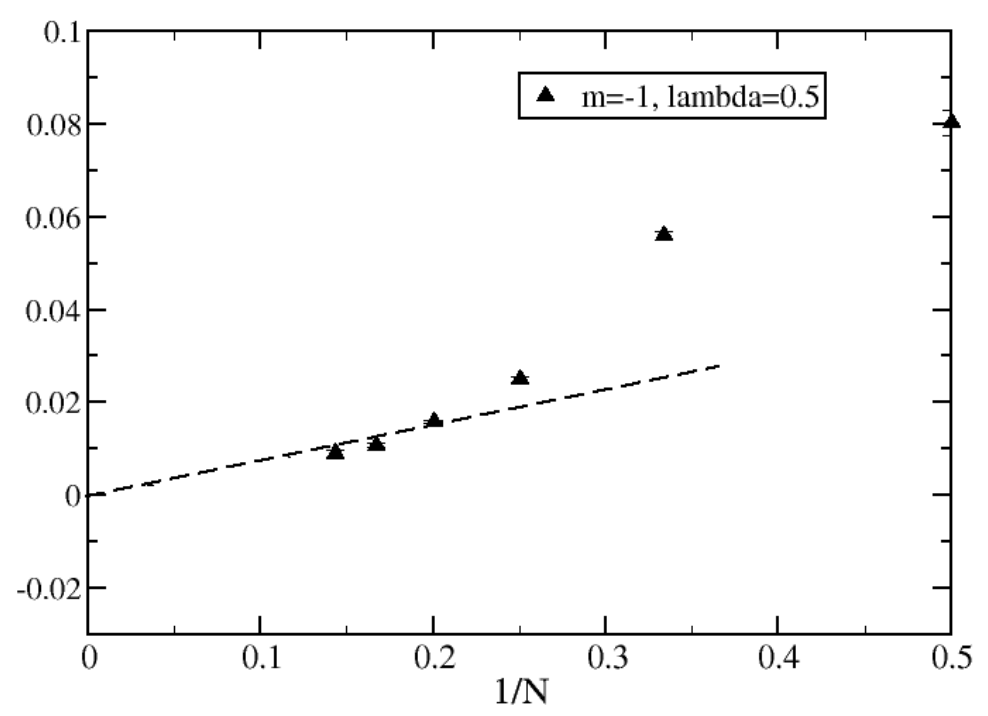

Figure 5. Polyakov line vs $1 / N$ for $\lambda=0.5$ and $m=-1$.

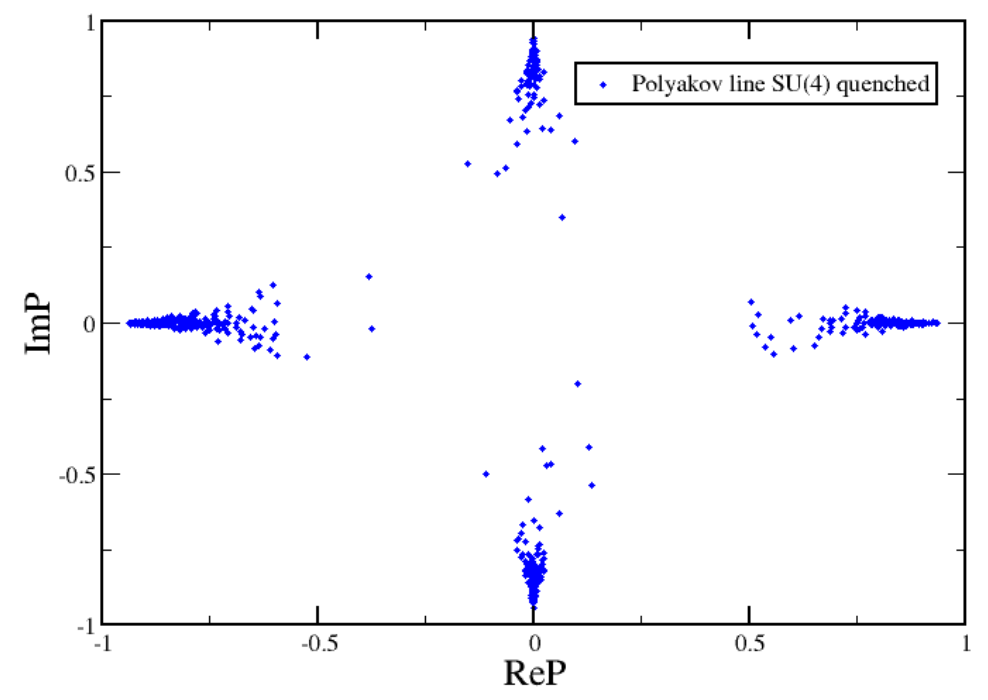

Figure 6. Scatter plot of values of $P$ for quenched $\mathrm{SU}(4) \lambda=0.5$.

bare fermion mass $m=-1$ placing it close to the critical line). We show data in figures 6 and 7 for the case $N=4$. The quenched data shows quite clearly the effect of symmetry breaking - the values of $P$ cluster about 4 points in the complex plane corresponding to the eigenvalues of $P$ localizing on the fourth roots of unity as expected in the broken phase. The dynamical runs show a symmetric clustering of values around the origin as the corresponding eigenvalues spread uniformly around the unit circle and provides strong evidence for a restoration of center symmetry. One might worry that the data we have shown so far indeed indicates that center symmetry is at least partially realized in the large $N$ limit but does not exclude the possibility of more exotic breakings in which, for 


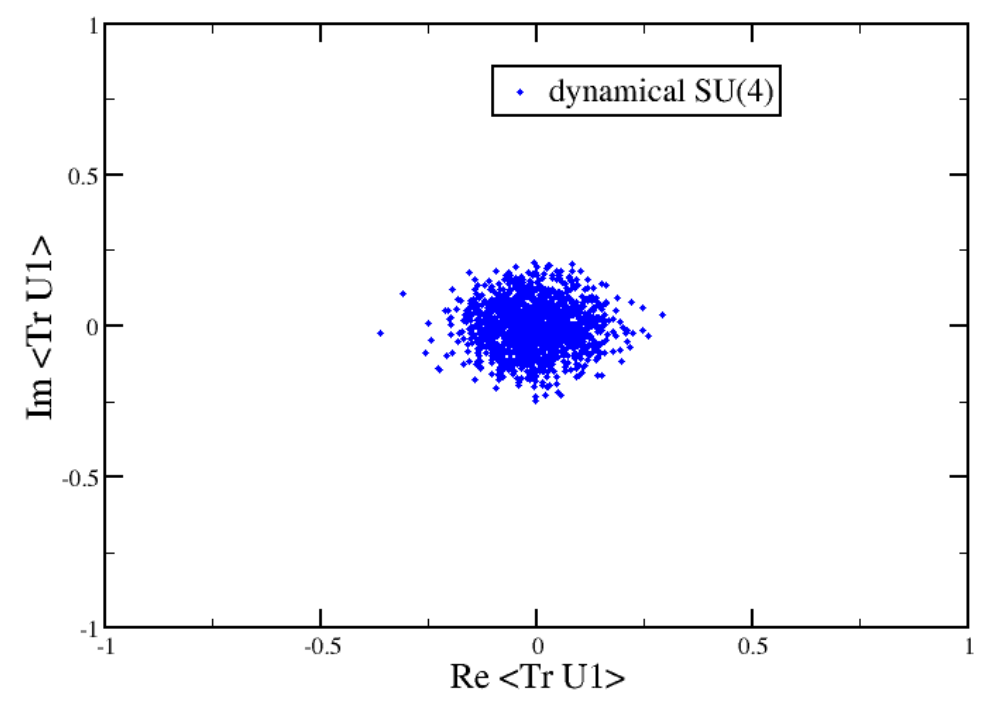

Figure 7. Scatter plot of values of $P$ for dynamical SU(4) $\lambda=0.5$.

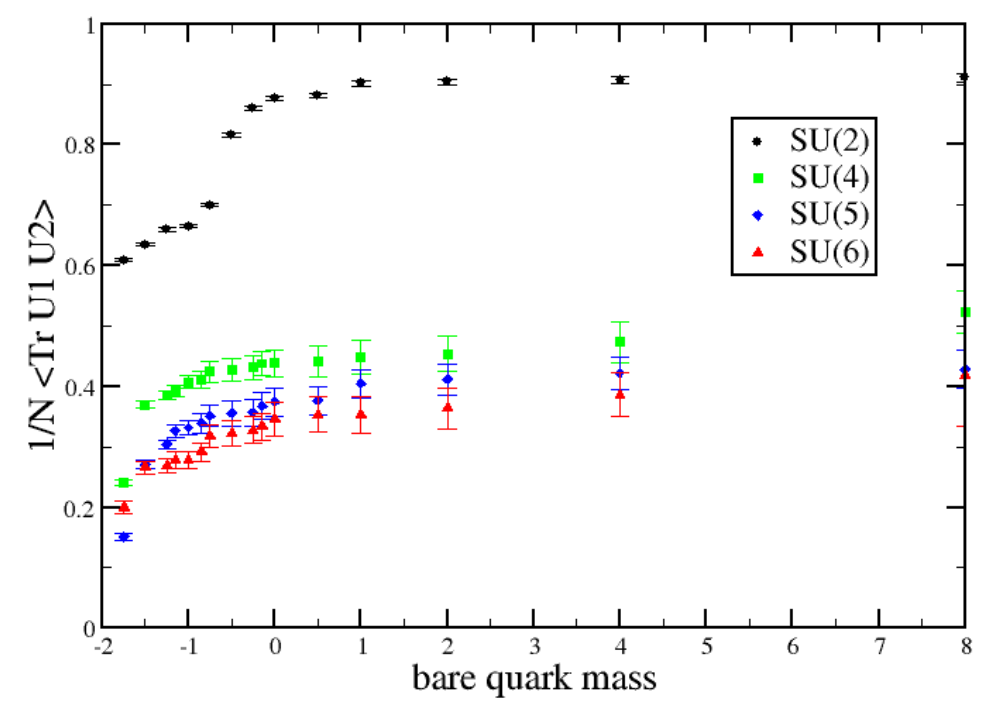

Figure 8. $M^{(1)}$ vs bare quark mass for 't Hooft coupling $\lambda=0.5$.

example, the expectation value of the Polyakov line is vanishing in any particular direction but that other correlators such as $M_{\mu \nu}^{(1)}$ or $M_{\mu \nu}^{(2)}$ are non-zero - corresponding to a locking of the values of the lines in different directions. We find that this is not the case; figure 8 shows the value of $\frac{1}{N}\left|\operatorname{Tr}\left(M_{12}^{(1)}\right)\right|$ versus bare quark mass for a range of $N$ and figure 9 similar curves for $\frac{1}{N}\left|\operatorname{Tr}\left(M_{12}^{(2)}\right)\right|$. These plots show results which are consistent with a full realization of center symmetry for light quarks in the large $N$ limit in agreement with the perturbative analysis. 


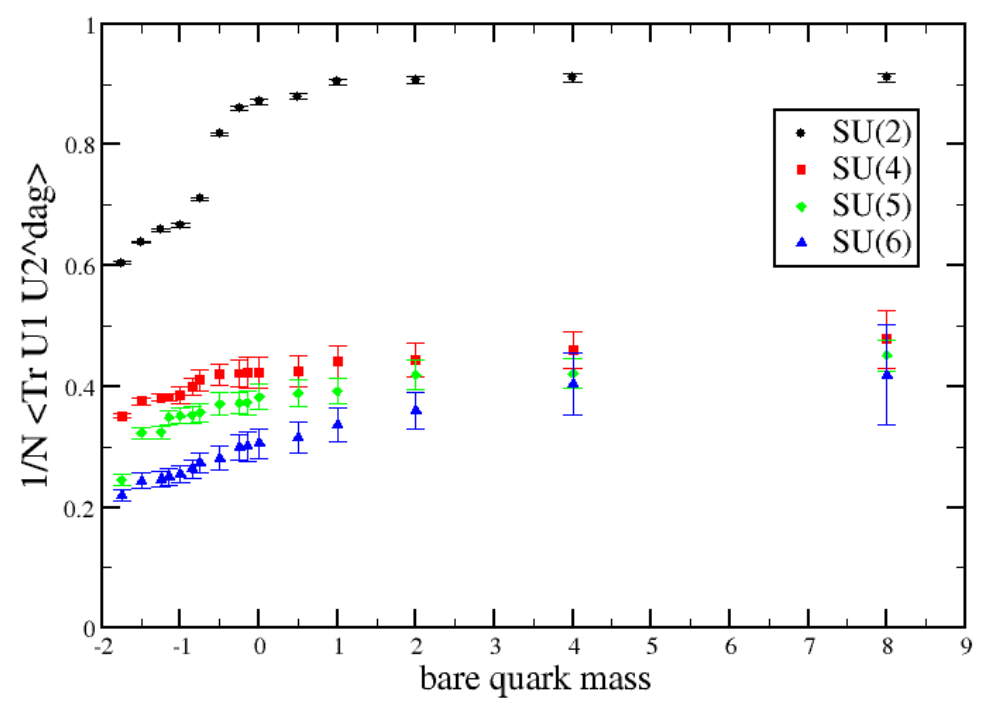

Figure 9. $M^{(2)}$ vs bare quark mass for 't Hooft coupling $\lambda=0.5$.

\section{Conclusions}

Our analytical and numerical results suggest that the vacuum of $\mathrm{SU}(N)$ gauge theory with two flavors of light adjoint Wilson fermion realizes full center symmetry in the large $N$ limit. This opens up the possibility of studying the (near) conformal behavior of the SU(2) minimal walking theory by examining the large- $N$ behavior of its $\mathrm{SU}(N)$ generalization on small lattices. We hope this will give a useful additional theoretical tool for determining whether this theory does indeed possess an infrared conformal fixed point.

\section{Acknowledgments}

S.M.C. is supported in part by DOE grant DE-FG02-85ER40237. The simulations were carried out using USQCD resources at Fermilab. R.G. would like to thank Ari Hietanen for useful discussions and Syracuse University for the award of a STEM graduate fellowship. M.Ü's work is supported by the U.S. Department of Energy Grant DE-AC02-76SF00515.

Open Access. This article is distributed under the terms of the Creative Commons Attribution Noncommercial License which permits any noncommercial use, distribution, and reproduction in any medium, provided the original author(s) and source are credited.

\section{References}

[1] T. Eguchi and H. Kawai, Reduction of Dynamical Degrees of Freedom in the Large- $N$ Gauge Theory, Phys. Rev. Lett. 48 (1982) 1063 [SPIRES].

[2] L.G. Yaffe, Large-N Limits as Classical Mechanics, Rev. Mod. Phys. 54 (1982) 407 [SPIRES]. 
[3] G. Bhanot, U.M. Heller and H. Neuberger, The Quenched Eguchi-Kawai Model, Phys. Lett. B 113 (1982) 47 [SPIRES].

[4] A. Gonzalez-Arroyo and M. Okawa, The Twisted Eguchi-Kawai Model: A Reduced Model for Large-N Lattice Gauge Theory, Phys. Rev. D 27 (1983) 2397 [SPIRES].

[5] P. Kovtun, M. Ünsal and L.G. Yaffe, Volume independence in large- $N_{c}$ QCD-like gauge theories, JHEP 06 (2007) 019 [hep-th/0702021] [SPIRES].

[6] M. Ünsal and L.G. Yaffe, Large- $N$ volume independence in conformal and confining gauge theories, arXiv:1006.2101 [SPIRES].

[7] E. Poppitz and M. Ünsal, AdS/CFT and large-N volume independence, arXiv:1005.3519 [SPIRES].

[8] F. Sannino and K. Tuominen, Orientifold theory dynamics and symmetry breaking, Phys. Rev. D 71 (2005) 051901 [hep-ph/0405209] [SPIRES].

[9] D.D. Dietrich and F. Sannino, Walking in the SU(N), Phys. Rev. D 75 (2007) 085018 [hep-ph/0611341] [SPIRES].

[10] N. Evans and F. Sannino, Minimal walking technicolour, the top mass and precision electroweak measurements, hep-ph/0512080 [SPIRES].

[11] D.D. Dietrich, F. Sannino and K. Tuominen, Light composite Higgs from higher representations versus electroweak precision measurements: Predictions for $\mathrm{LHC}$, Phys. Rev. D 72 (2005) 055001 [hep-ph/0505059] [SPIRES].

[12] R. Foadi, M.T. Frandsen, T.A. Ryttov and F. Sannino, Minimal Walking Technicolor: Set Up for Collider Physics, Phys. Rev. D 76 (2007) 055005 [arXiv:0706. 1696] [SPIRES].

[13] M. Antola, S. Di Chiara, F. Sannino and K. Tuominen, Minimal Super Technicolor, arXiv: 1001.2040 [SPIRES].

[14] S. Catterall and F. Sannino, Minimal walking on the lattice, Phys. Rev. D 76 (2007) 034504 [arXiv: 0705.1664] [SPIRES].

[15] S. Catterall, J. Giedt, F. Sannino and J. Schneible, Phase diagram of SU(2) with 2 flavors of dynamical adjoint quarks, JHEP 11 (2008) 009 [arXiv:0807.0792] [SPIRES].

[16] L. Del Debbio, B. Lucini, A. Patella, C. Pica and A. Rago, The infrared dynamics of Minimal Walking Technicolor, arXiv:1004.3206 [SPIRES].

[17] L. Del Debbio, B. Lucini, A. Patella, C. Pica and A. Rago, Mesonic spectroscopy of Minimal Walking Technicolor, arXiv:1004.3197 [SPIRES].

[18] L. Del Debbio, B. Lucini, A. Patella, C. Pica and A. Rago, Conformal vs confining scenario in SU(2) with adjoint fermions, Phys. Rev. D 80 (2009) 074507 [arXiv:0907.3896] [SPIRES].

[19] F. Bursa, L. Del Debbio, L. Keegan, C. Pica and T. Pickup, Mass anomalous dimension in SU(2) with two adjoint fermions, Phys. Rev. D 81 (2010) 014505 [arXiv:0910.4535] [SPIRES].

[20] F. Bursa, L. Del Debbio, L. Keegan, C. Pica and T. Pickup, Running of the coupling and quark mass in SU(2) with two adjoint fermions, arXiv:0910.2562 [SPIRES].

[21] A. Hietanen, J. Rantaharju, K. Rummukainen and K. Tuominen, Minimal technicolor on the lattice, Nucl. Phys. A $\mathbf{8 2 0}$ (2009) 191c-194c [SPIRES]. 
[22] A.J. Hietanen, K. Rummukainen and K. Tuominen, Evolution of the coupling constant in SU(2) lattice gauge theory with two adjoint fermions, Phys. Rev. D 80 (2009) 094504 [arXiv:0904.0864] [SPIRES].

[23] S. Catterall, J. Giedt, F. Sannino and J. Schneible, Probes of nearly conformal behavior in lattice simulations of minimal walking technicolor, arXiv:0910.4387 [SPIRES].

[24] J. Kiskis, R. Narayanan and H. Neuberger, Does the crossover from perturbative to nonperturbative physics in $Q C D$ become a phase transition at infinite $N$ ?, Phys. Lett. B 574 (2003) 65 [hep-lat/0308033] [SPIRES].

[25] M. Ünsal and L.G. Yaffe, Center-stabilized Yang-Mills theory: confinement and large-N volume independence, Phys. Rev. D 78 (2008) 065035 [arXiv:0803.0344] [SPIRES].

[26] B. Bringoltz and S.R. Sharpe, Non-perturbative volume-reduction of large- $N$ QCD with adjoint fermions, Phys. Rev. D 80 (2009) 065031 [arXiv:0906.3538] [SPIRES].

[27] W. Bietenholz, J. Nishimura, Y. Susaki and J. Volkholz, A non-perturbative study of $4 D$ U(1) non-commutative gauge theory: The fate of one-loop instability, JHEP 10 (2006) 042 [hep-th/0608072] [SPIRES].

[28] M. Teper and H. Vairinhos, Symmetry breaking In twisted Eguchi-Kawai models, Phys. Lett. B 652 (2007) 359 [hep-th/0612097] [SPIRES].

[29] T. Azeyanagi, M. Hanada, T. Hirata and T. Ishikawa, Phase structure of twisted Eguchi-Kawai model, JHEP 01 (2008) 025 [arXiv:0711.1925] [SPIRES].

[30] B. Bringoltz and S.R. Sharpe, Breakdown of large- $N$ quenched reduction in SU(N) lattice gauge theories, Phys. Rev. D 78 (2008) 034507 [arXiv:0805.2146] [SPIRES].

[31] G. Cossu and M. D'Elia, Finite size phase transitions in QCD with adjoint fermions, JHEP 07 (2009) 048 [arXiv: 0904.1353] [SPIRES].

[32] P.F. Bedaque, M.I. Buchoff, A. Cherman and R.P. Springer, Can fermions save large- $N$ dimensional reduction?, JHEP 10 (2009) 070 [arXiv:0904.0277] [SPIRES].

[33] B. Bringoltz, Large- $N$ volume reduction of lattice $Q C D$ with adjoint Wilson fermions at weak-coupling, JHEP 06 (2009) 091 [arXiv: 0905.2406] [SPIRES].

[34] B. Bringoltz, Partial breakdown of center symmetry in large- $N$ QCD with adjoint Wilson fermions, JHEP 01 (2010) 069 [arXiv:0911.0352] [SPIRES].

[35] E. Poppitz and M. Ünsal, Comments on large-N volume independence, JHEP 01 (2010) 098 [arXiv:0911.0358] [SPIRES].

[36] J.C. Myers and M.C. Ogilvie, Exotic phases of finite temperature SU(N) gauge theories, Nucl. Phys. A 820 (2009) 187c-190c [arXiv:0810.2266] [SPIRES].

[37] P.N. Meisinger and M.C. Ogilvie, String Tension Scaling in High-Temperature Confined SU(N) Gauge Theories, Phys. Rev. D 81 (2010) 025012 [arXiv:0905.3577] [SPIRES].

[38] A. Hietanen and R. Narayanan, The large- $N$ limit of four dimensional Yang-Mills field coupled to adjoint fermions on a single site lattice, arXiv:0911.2449 [SPIRES].

[39] T. Azeyanagi, M. Hanada, M. Ünsal and R. Yacoby, Large- $N$ reduction in QCD-like theories with massive adjoint fermions, arXiv:1006.0717 [SPIRES].

[40] S. Duane, A. Kennedy, B. Pendleton and D. Roweth, Hybrid Monte Carlo, Phys. Lett. B 195 (1987) 216. 